\title{
Prognostic role of RDW in hematological malignancies: a systematic review and meta-analysis
}

Lisha $\mathrm{Ai}^{*}$, Shidai $\mathrm{Mu}^{\dagger}$ and $\mathrm{Yu} \mathrm{Hu}{ }^{*}$

\begin{abstract}
Background: Red blood cell distribution width (RDW), a biomarker for discrimination of anemia, has been recently identified as a prognostic factor in various types of cancer. Here we performed a meta-analysis in order to assess the correlation between RDW and the survival outcomes in patients with hematologic malignances.

Patients/methods: We systematically searched PubMed, Embase, and ISI Web of Science for relevant studies, to investigate the prognostic significance of RDW in hematological malignancies. Odds ratios or hazards ratios (HRs) with corresponding 95\% confidence intervals (Cls) are pooled to estimate the association between RDW and clinicopathological parameters of patients with hematologic malignances.

Results: Seven trials with 1031 patients suffering from hematological malignancies were included in the metaanalysis, and the results indicated that increased pretreatment RDW predicted poor overall survival $(\mathrm{HR}=2.35,95 \% \mathrm{Cl}$ 1.70-3.24), poor progress-free survival ( $H R=2.44,95 \% \mathrm{Cl} 1.70-3.49)$ and poor event-free survival (EFS) $(H R=3.15,95 \%$ Cl 1.59-6.25). Furthermore, the similar results were observed in subgroup analysis stratified by cancer type, such as multiple myeloma, and diffuse large B cell lymphoma, etc.
\end{abstract}

Conclusions: As for hematologic malignances, patients with higher RDW are more likely to have poorer prognosis than those with lower RDW.

Keywords: Red blood cell distribution width, Hematologic malignances, Prognosis, Meta-analysis

\section{Background}

Hematological malignancies mainly include leukemia, lymphoma, and plasma cell neoplasm. There were about 172,910 new cases of hematological malignancies and 58,300 deaths due to hematological malignancies projected to occur in 2017 in USA [1]. Great advances have recently been achieved in the therapy for patients with hematologic malignances. However, the overall survival for patients has not been obviously improved. Identification of prognostic factors for hematologic malignancies is very helpful for clinicians to choose therapeutic strategies and for patients to improve their prognosis.

*Correspondence: ailisha@hust.edu.cn; dr_huyu@126.com ${ }^{\dagger}$ Lisha $\mathrm{Ai}$ and Shidai Mu contributed equally to this work Institute of Hematology, Union Hospital, Tongji Medical College, Huazhong University of Science and Technology, Wuhan 430022, China
A number of prognostic molecular markers for hematologic malignances have been identified, however, many of these prognostic means are costly, difficult to perform, or not easily interpreted. Therefore, other prognostic models that are inexpensive, widely available, and easily interpreted are urgently needed for clinicians.

Red blood cell distribution width (RDW) is a parameter measured in blood routine test, and is widely used to distinguish between different types of anemia [2]. As an easy-to-measure marker of the systemic inflammatory response, the RDW has been established as a novel prognostic factor in many pathophysiological conditions, including cardiovascular disease [3, 4] and inflammation [5, 6]. Recently, RDW grows to be recognized as an independent prognosis factor in numerous types of cancer, including lung cancer [7], gastrocolorectal cancer [8], 
breast cancer [9], prostate cancer [10], as well as in several types of hematologic malignances.

However, due to the variance in the study design and sample size, direct impact of RDW level on hematologic malignances patients' survival remains inconclusive. In this study, we searched PubMed (Medline), OVID (Embase), and ISI Web of Science databases for relevant studies and performed a meta-analysis in order to assess the correlation between RDW and the survival outcomes in patients with hematologic malignances.

\section{Methods}

\section{Search strategy}

We conducted the systematic search strategies described by Dickersin et al. [11] to identify all relevant electric publications until January 2018 throughout databases, including (Medline), OVID (Embase), and ISI Web of Science databases. The search strategy included terms are as follow: "RDW" (e.g. "red blood cell distribution width"), "prognosis" (e.g. "outcome" "survival" "mortality" "recurrence" "progression" "metastasis") and "hematologic malignancies" (e.g. "leukemia" "lymphoma" "myeloma" "myelodysplastic syndromes"). Furthermore, we manually checked the reference lists of retrieved studies to identify more potential pertinent studies.

\section{Selection criteria}

Studies were included in the meta analysis if they met all of the following criteria: (i) patients were diagnosed with hematologic malignancies; (ii) association between the pretreatment RDW and OS, PFS or other clinicopathological parameters was reported; (iii) studies that were not directly reporting hazard ratios (HRs) and 95\% CI were allowed if we could reconstruct them by $p$ values and other data reported [12]; (iv) the publication language was confined to English. Exclusion criteria were: (i) abstracts, letters, reviews, case reports, etc.; (ii) studies with insufficient data for analysis; (iii) studies without specific data concerning hematologic malignancies or RDW; (iv) multiple published reports. When there were several reports concerning the same cohort, we included the most recent publication in our meta-analysis.

\section{Data extraction}

Two investigators (Lisha $\mathrm{Ai}$ and Shidai $\mathrm{Mu}$ ) independently identified the eligible studies for this metaanalysis. Any disagreement was resolved by discussion with the other researcher ( $\mathrm{Yu} \mathrm{Hu}$ ). The qualities of the included studies were assessed according to the Newcastle-Ottawa Quality Assessment Scale (NOS) [13]. This scale uses a star system (with a maximum of nine stars) to evaluate a study in three domains: selection of participants, comparability of study groups, and the ascertainment of outcomes of interest. NOS scores of $\geq 7$ were assigned as high-quality studies.

For each study, the following relevant data were extracted in a predefined table: (i) first author's name, year of publication, country of the population, sample size, patient age, follow-up period; (ii) survival data including overall survival (OS), progression free survival (PFS) and event free survival (EFS) (OS was calculated from the medical treatment until the death of patient or the last follow-up. PFS was defined as the interval between the date of treatment and the detection of the recurrence tumor or death from any cause. EFS was calculated from the first day of diagnosis until any events, such as disease progression or relapse, initiation of another treatment, death due to any cause, etc.); Get Data Graph Digitizer (http://getdata-graph-digitizer. $\mathrm{com} /$ ) were used to obtain the data from the survival curve. (iii) cut-off value used to define "elevated RDW".

\section{Statistical analysis}

Hazard ratio (HR) and 95\% confidence intervals (95\% $\mathrm{CI})$ were obtained directly from each literature or from estimation according to the methods by Parmer et al. [12]. Heterogeneity among included studies was checked by the $X^{2}$-based $Q$ test and $I^{2}$ test [14]. The fixed-effect model was used for analysis without any significant heterogeneity between studies $\left(p>0.10, \mathrm{I}^{2}<50 \%\right)$. Otherwise, the random-effects model was chosen. Subgroup analysis was further performed to explore the source of heterogeneity. Sensitivity analysis was also performed to examine the effect of each study on the overall pooled results. Publication bias was evaluated by using Begg's test and Egger's test. Trim-and-fill method was employed to further assess the possible effect of publication bias [39]. All analyses were carried out using STATA statistical software package version 12.0 (STATA, College Station, TX).

\section{Results \\ Selection and characteristics of included studies}

As shown in Fig. 1, the initial search algorithm retrieved a total of 145 studies. After excluding the duplicates $(\mathrm{n}=45)$; abstracts, letters, reviews, etc. $(\mathrm{n}=9)$; and the studies not related to research topics $(n=66)$, the remaining studies $(n=25)$ were further reviewed by reading the full text. Additional 18 studies were then excluded because they didn't provide specific data concerning hematologic malignances nor RDW. Therefore, 7 studies between 2014 and 2017 with a total 1031 hematologic malignances patients were enrolled in our meta-analysis.

Summary on the characteristics of the included studies were shown in Table 1. These studies were from China, Japan, Korea and Croatia, which evaluated several type of 

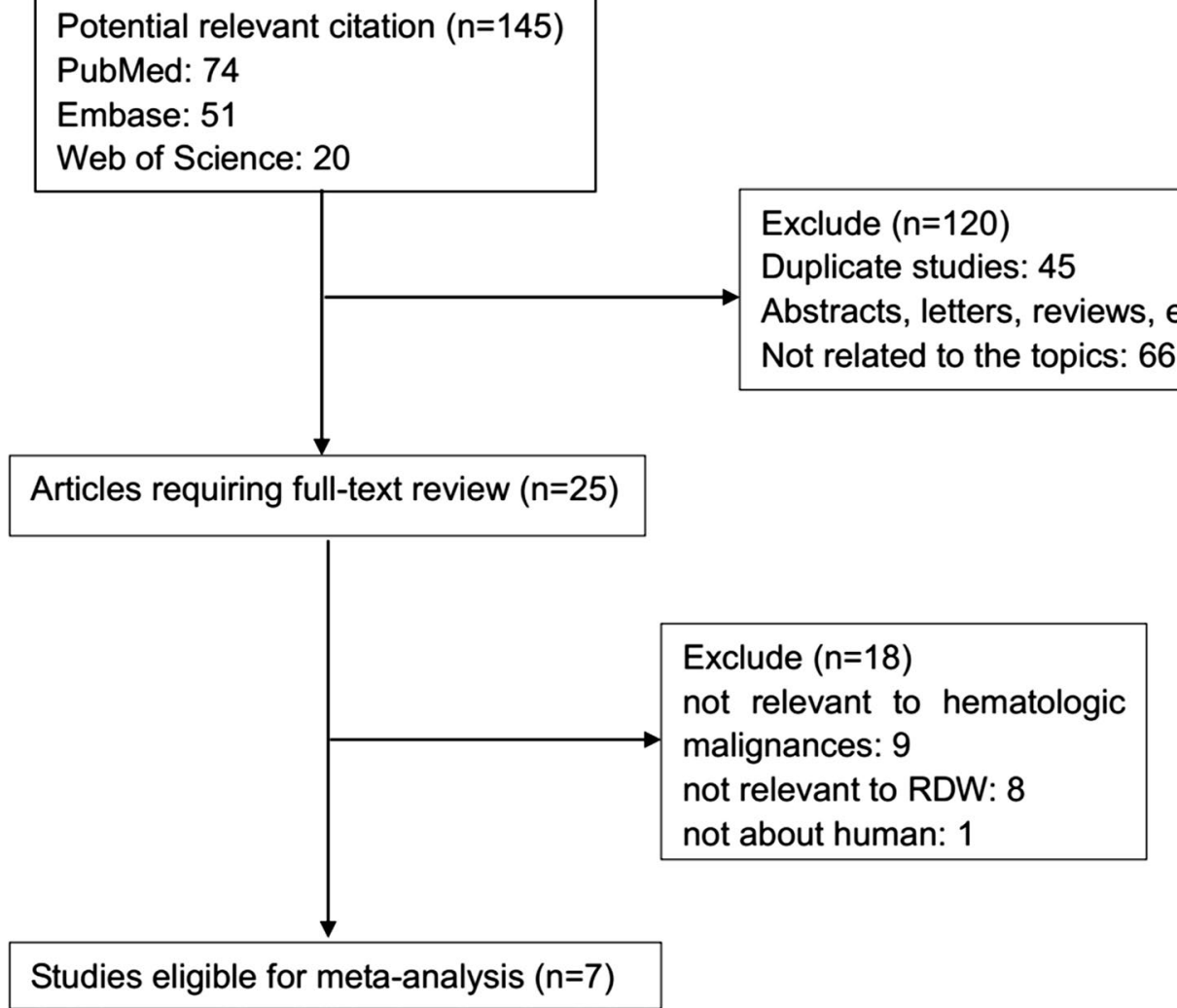

Fig. 1 Flow diagram of selecting relevant studies included in the meta-analysis

Table 1 Characteristics of studies included in the meta-analysis

\begin{tabular}{|c|c|c|c|c|c|c|c|c|c|c|}
\hline Study & Year & Country & Cancer types & Sample size & Cut-off & Age & Follow-up (month) & Survival analysis & HR & NOS score \\
\hline Zhou & 2017 & China & DLBCL & 161 & 14.1 & $59(18-80)$ & $42(6-120)$ & OS, PFS & Reported & 7 \\
\hline Wang & 2017 & China & MM & 196 & 18.05 & $65(33-82)$ & $33.5(1-120)$ & OS & Reported & 7 \\
\hline Meng & 2017 & China & MM & 166 & 14 & 61.6 & $17.79(0.63-62.83)$ & OS, PFS & Reported & 4 \\
\hline Luo & 2017 & China & NK/T lymphoma & 191 & $46.2^{\mathrm{a}}$ & $44(15-86)$ & $30(2-97)$ & OS, PFS & Reported & 7 \\
\hline Perisa & 2015 & Croatia & DLBCL & 81 & 15 & 64 & NR & OS, EFS & Reported & 5 \\
\hline Iriyama & 2015 & Japan & CML & 90 & 15 & $N R$ & 168 & OS, EFS & Estimated & 5 \\
\hline Lee & 2014 & Korea & MM & 146 & 14.5 & $61(32-83)$ & 120 & PFS & Reported & 6 \\
\hline
\end{tabular}

NR not reported, NOS Newcastle-Ottawa Quality Assessment Scale

a RDW was present as RDW-SD

hematologic malignancies, including three for multiple myeloma (MM), two for diffuse large B cell lymphoma (DLBCL), one for extranodal NK/T lymphoma (ENKT), and one for chronic myeloid leukemia (CML). Five studies enrolled $>100$ patients and two studies had $<100$ patients. The cutoff value defining high RDW in these studies was not uniform and ranged from 14.0 to 18.05. One study used RDW-SD (standard deviation) for RDW and others used RDW-CV (covariance). 885 patients from six studies reported OS, 664 patients from four studies reported PFS and 171 patients from two studies reported EFS. Six studies directly reported HR and 95\% $\mathrm{CI}$ in the original literature. NOS score was above 7 in 4 studies.

\section{Association between RDW and survival of hematologic malignances patients}

7 studies in our analysis examined the association between RDW and survival of patients with hematologic malignances. As shown in Fig. 2, the combined 
results of 7 studies showed elevated RDW was associated with poor $\mathrm{OS}(\mathrm{HR}=2.35,95 \% \mathrm{CI} 1.70-3.24)$ without significant heterogeneity $\left(\mathrm{I}^{2}=0 \%, \mathrm{P}_{\text {heterogeneity }}=0.566\right)$. Figure 3 summarized $\mathrm{HR}$ for $\mathrm{PFS}(\mathrm{HR}=2.44,95 \% \mathrm{CI}$ $1.70-3.49)$ and EFS (HR=3.15, 95\% CI 1.59-6.25), and there were no heterogeneity between the studies $\left(\mathrm{I}^{2}=0 \%\right.$, $\mathrm{P}_{\text {heterogeneity }}=0.725 ; \quad$ and $\mathrm{I}^{2}=0 \%, \mathrm{P}_{\text {heterogeneity }}=0.573$, respectively).

Subgroup analysis for OS was also performed stratified by cancer type. As shown in Fig. 4, summarized HR for DLBCL $(\mathrm{HR}=3.18,95 \%$ CI $1.85-5.45)$, MM $(\mathrm{HR}=1.70$, 95\% CI $0.94-3.09)$ and other types $(\mathrm{HR}=2.26,95 \% \mathrm{CI}$ 1.32-3.87), and there was no heterogeneity between the studies $\left(\mathrm{I}^{2}=0 \%, \mathrm{P}_{\text {heterogeneity }}=0.793 ; \mathrm{I}^{2}=0 \%\right.$, $\mathrm{P}_{\text {heterogeneity }}=0.326 ; \quad$ and $\mathrm{I}^{2}=0 \%, \quad \mathrm{P}_{\text {heterogeneity }}=0.478$, respectively).

\section{Sensitivity analysis}

Sensitivity analyses were performed next. A single study involved in the meta-analysis was deleted each time to unveil the influence of the individual data set on the pooled HRs. As shown in Fig. 5, there was no study obviously impacting the combined results, which indicated the robustness of our meta-analytic results.

\section{Publication bias}

To assess publication bias in this study, the included studies were conducted by using Begg's funnel plots and Egger's test. The results indicated the possibility of publication bias among the studies included in our analysis $(p=0.481)$. Therefore, "trim and fill" analysis was further performed, and the result indicated that one relevant study evaluating the prognostic value of elevated RDW in hematologic malignances patients remained unpublished. However, the pooled HR of 2.27 (95\% CI 1.663.09) obtained from trim and fill method was statistically significant with a symmetrical funnel plot (Fig. 6).

\section{Discussion}

Cancer associated inflammation is recognized as a hallmark feature of tumor development and progression. Previous studies have reported the association between RDW and the clinical outcome of solid tumor. Recently, numerous studies have provided evidence on the correlation between elevated RDW and poor prognosis in hematologic malignances, including chronic lymphocytic leukemia (CLL) [15], chronic myeloid leukemia (CML) [16], DLBCL [17, 18], NK/T lymphoma [19], as well as multiple myeloma [20-22].

Study
ID




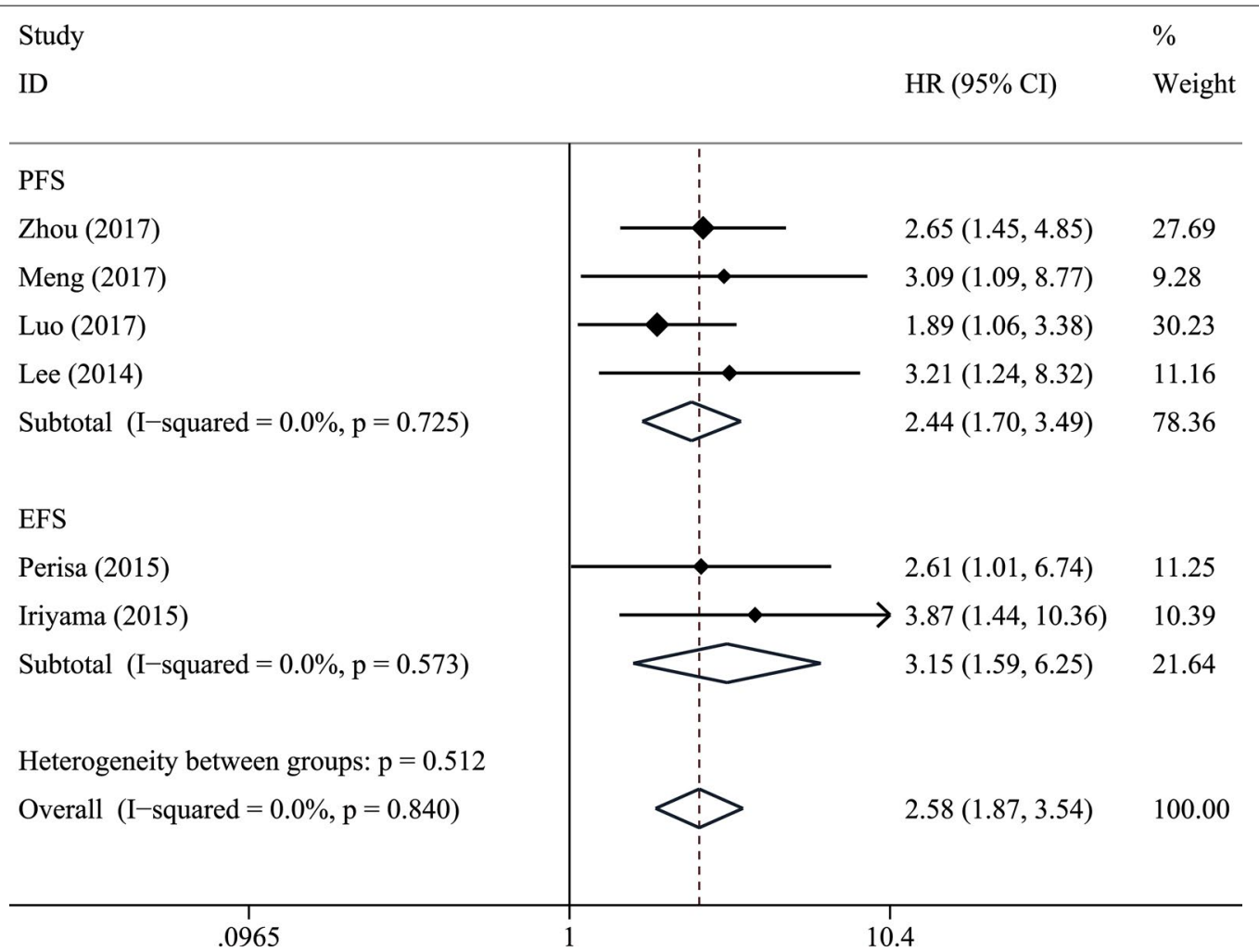

Fig. 3 Forest plot for the association between elevated RDW and PFS and EFS in hematologic malignances

However, these results are not comparable, because of the heterogeneous designs and patient population, and the diversity in cut-off value defining "elevated RDW". Our study is the first meta-analysis covering a total of 7 published studies with 1031 patients to clarify the prognostic value of RDW in the pretreatment patients with hematologic malignances. The combined results indicated that elevated RDW significantly predicted poor OS, poor EFS and poor PFS of patients with hematologic malignances. Furthermore, the similar results were observed in subgroup analysis stratified by cancer type, such as MM, DLBCL, etc.

The prognostic value of RDW was investigated in a variety of cancer patients and gathering evidences suggested that RDW was an independent factor for prognosis [23, 24]. The exact mechanism underlying the associations of RDW with these cancers has not been clearly elucidated. Recently, numerous studies have reported the positive correlation between RDW and a variety of inflammatory markers, including the erythrocyte sedimentation rate (ESR), interleukin-6 (IL-6), C-reactive protein (CRP), soluble tumor necrosis factor (TNF) receptors I and II, and soluble transferrin receptor [25]. One possible explanation for this finding is that inflammation impairs erythropoiesis and causes changes in red blood cell maturation, which contributed to the increase in RDW [26]. In addition, RDW was found to be associated with malnutrition (i.e., deficiencies in nutrients such as vitamin B12 and folate), which has been shown to be correlated to lower response to treatment, and poorer prognosis in cancer patients [18]. Moreover, in the terminal stage of malignancy, digestive system dysfunction may lead to inadequate resorption of the iron, resulting in the disturbed iron metabolism and the inhibition of iron transport in the blood, which might contribute to increased RDW levels. This mechanism has been found in most of the cancers [27]. Therefore, elevated RDW might bridge the relationship between inflammation and tumorigenesis, thereby correlating to poor prognosis of cancer patients.

This meta-analysis had some limitations that call for cautious interpretation of the results. First, only 7 studies were included in this meta-analysis, and tumor types of this study were also limited, which could decrease the accuracy of the results. Second, the cut-off value defining elevated RDW varied among studies (Table 1). Third, differences of paper quality and sample size across the studies might cause bias in the meta-analysis. Forth, most of the included studies reported positive results, therefore our results might overestimate the prognostic significance of RDW to some degree. 


\section{Study}

ID

DLBCL

Zhou (2017)

Perisa (2015)

Subtotal $(\mathrm{I}-$ squared $=0.0 \%, \mathrm{p}=0.793)$

MM

Wang (2017)

Meng (2017)

Subtotal $(\mathrm{I}-$ squared $=0.0 \%, \mathrm{p}=0.326)$

Other

Luo (2017)

Iriyama (2015)

Subtotal $(\mathrm{I}-$ squared $=0.0 \%, \mathrm{p}=0.478)$

Heterogeneity between groups: $\mathrm{p}=\mathbf{0 . 3 0 9}$

Overall $(\mathrm{I}-$ squared $=0.0 \%, \mathrm{p}=0.566)$
HR $(95 \%$ CI $)$

$\%$

Weight

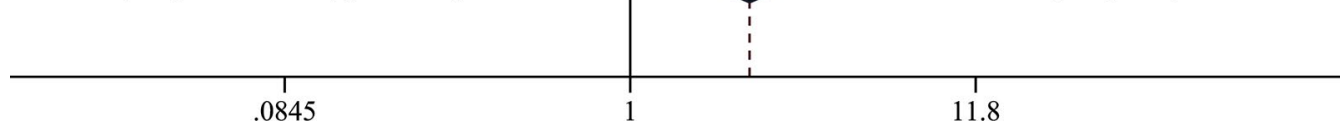

$2.04(1.12,3.75) \quad 28.09$

$3.29(1.02,10.63) \quad 7.51$

$2.26(1.32,3.87) \quad 35.59$

$1.38(0.67,2.86) \quad 19.54$

$2.62(0.92,7.46) \quad 9.41$

$1.70(0.94,3.09) \quad 28.95$

27.99

$3.18(1.85,5.45) \quad 35.45$

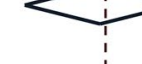

Fig. 4 Forest plot for the association between RDW and OS in different types of hematologic malignances

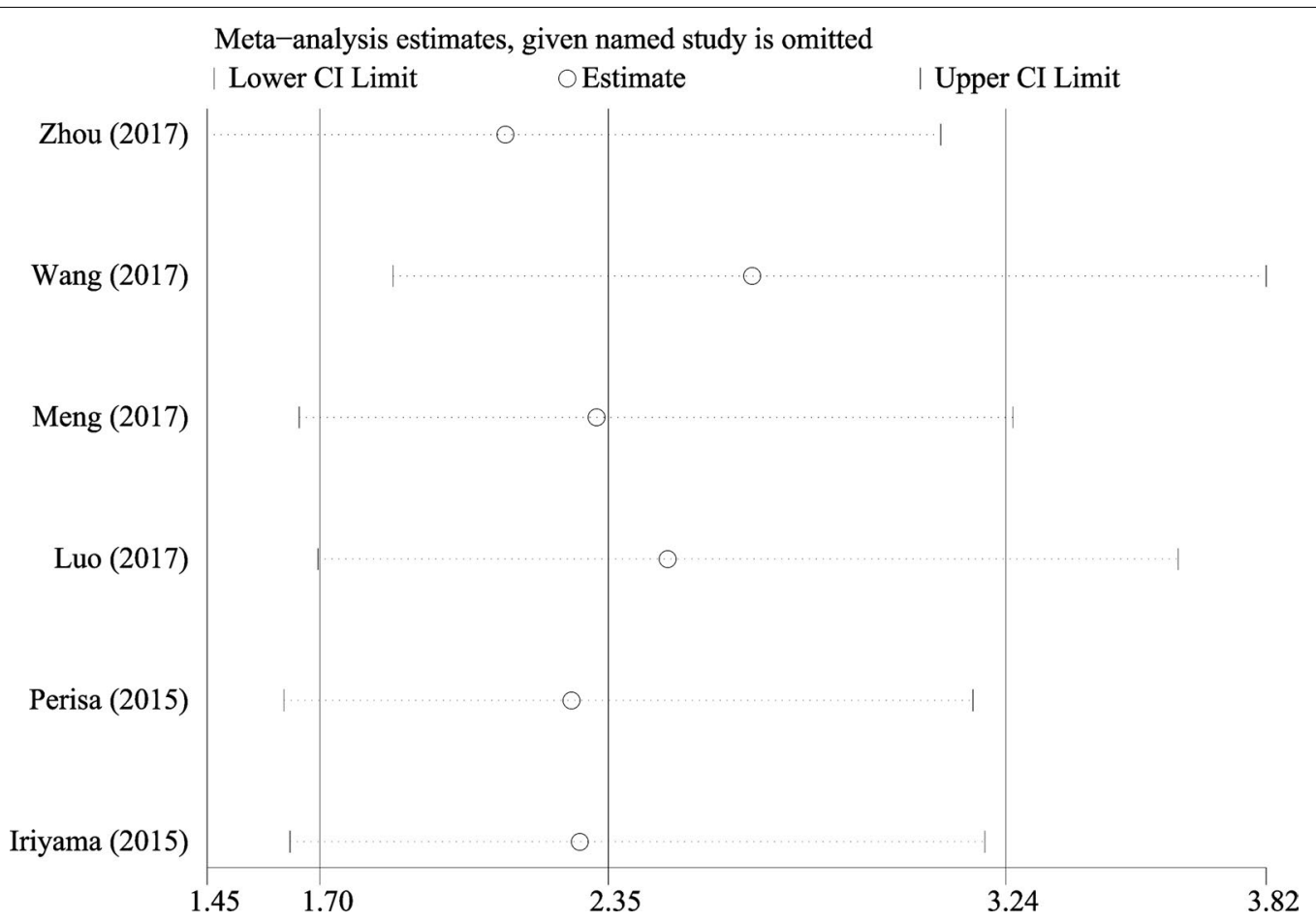

Fig. 5 Sensitivity analysis of the enrolled analysis 


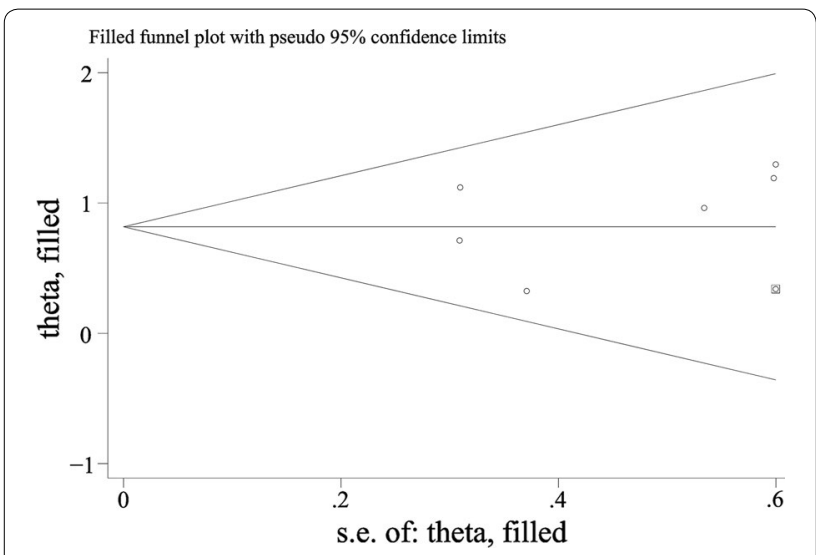

Fig. 6 Funnel plot with trim and fill

Despite the above limitations, our meta-analysis supports the values of RDW for predicting survival outcome in patients of hematologic malignances. RDW can be easily obtained from routine blood tests, thus intermediate assessments about changes in RDW during therapy were simply available. That is, RDW can help personalize the treatment intensity, as well as aftercare schedule, in order to increase the likelihood of early detection.

\section{Conclusion}

Here, we searched electronic databases for relevant studies, and enrolled 7 studies with a total of 1031 patients for meta-analysis, drawing a conclusion that patients with higher RDW are more likely to have poorer prognosis than those with lower RDW. Taken together, the results from our meta-analysis suggest that RDW gains a prognostic value for patients with hematologic malignances. More multi-center prospective cohorts should be conducted to further validate the role of the RDW in hematologic malignances.

\footnotetext{
Abbreviations

RDW: red blood cell distribution width; OR: odds ratio; HR: hazards ratios; $\mathrm{Cl}$ : confidence interval; OS: over survival; PFS: progress-free survival; EFS: event-free survival; NOS: Newcastle-Ottawa Quality Assessment Scale; MM: multiple myeloma; DLBCL: diffuse large B cell lymphoma; ENKT: extranodal NK/T lymphoma; CML: chronic myeloid leukemia; SD: standard deviation; CV: covariance; CLL: chronic lymphocytic leukemia; ESR: erythrocyte sedimentation rate; IL-6: interleukin-6; CRP: C-reactive protein;TNF: tumor necrosis factor.
}

\section{Authors' contributions}

SM and LA collected and analyzed the data, wrote the paper; $L A$ and $Y H$ conceived and designed this study, analyzed the data, wrote the paper; and all authors reviewed the paper. All authors read and approved the final manuscript.

\section{Acknowledgements}

We would like to thank the researchers and study participants for their contributions.

\section{Competing interests}

The authors declare that they have no competing interests.

Availability of data and materials

Please contact author for data requests.

Consent for publication

Not applicable.

\section{Ethics approval and consent to participate}

All procedures followed were in accordance with the ethical standards of the responsible committee on human experimentation (Research Ethics Committees of Wuhan Union Hospital, Tongji Medical College, Huazhong University of Science and Technology) and with the Helsinki Declaration of 1964 and later versions.

Funding

The National Natural Science Foundation of China (No. 81500172).

\section{Publisher's Note}

Springer Nature remains neutral with regard to jurisdictional claims in published maps and institutional affiliations.

Received: 19 March 2018 Accepted: 16 April 2018

Published online: 23 April 2018

\section{References}

1. Siegel RL, Miller KD, Jemal A. Cancer Statistics, 2017. CA Cancer J Clin. 2017:67(1):7-30.

2. Weiss G, Goodnough LT. Anemia of chronic disease. N Engl J Med. 2005:352(10):1011-23.

3. Mozos I. Mechanisms linking red blood cell disorders and cardiovascular diseases. Biomed Res Int 2015:2015:682054.

4. Arbel Y, Weitzman D, Raz R, Steinvil A, Zeltser D, Berliner S, Chodick G, Shalev V. Red blood cell distribution width and the risk of cardiovascular morbidity and all-cause mortality. A population-based study. Thromb Haemost. 2014:111(2):300-7.

5. Yesil A, Senates E, Bayoglu IV, Erdem ED, Demirtunc R, Kurdas Ovunc AO, Red cell distribution width: a novel marker of activity in inflammatory bowel disease. Gut Liver. 2011:5(4):460-7.

6. Jo YH, Kim K, Lee JH, Kang C, Kim T, Park HM, Kang KW, Kim J, Rhee JE. Red cell distribution width is a prognostic factor in severe sepsis and septic shock. Am J Emerg Med. 2013;31(3):545-8.

7. Koma Y, Onishi A, Matsuoka H, Oda N, Yokota N, Matsumoto Y, Koyama M, Okada N, Nakashima N, Masuya D, et al. Increased red blood cell distribution width associates with cancer stage and prognosis in patients with lung cancer. PLoS ONE. 2013;8(11):e80240.

8. Ay S, Eryilmaz MA, Aksoy N, Okus A, Unlu Y, Sevinc B. Is early detection of colon cancer possible with red blood cell distribution width? Asian Pac J Cancer Prev. 2015;16(2):753-6.

9. Seretis C, Seretis F, Lagoudianakis E, Gemenetzis G, Salemis NS. Is red cell distribution width a novel biomarker of breast cancer activity? Data from a pilot study. J Clin Med Res. 2013;5(2):121-6.

10. Albayrak S, Zengin $\mathrm{K}$, Tanik S, Bakirtas H, Imamoglu A, Gurdal M. Red cell distribution width as a predictor of prostate cancer progression. Asian Pac J Cancer Prev. 2014;15(18):7781-4.

11. Dickersin K, Scherer R, Lefebvre C. Identifying relevant studies for systematic reviews. BMJ (Clin Res Ed). 1994;309(6964):1286-91.

12. Parmar M, Torri V, Stewart L. Extracting summary statistics to perform meta-analysis of the published literature for survival endpoints. Stat Med. 1998;17:2815-34.

13. Wells G, Shea B, O'Connell D, Peterson j, Welch V, Losos M, Tugwell P. The Newcastle-Ottawa Scale (NOS) for assessing the quality of non-randomized studies in meta-analysis; 2000.

14. Higgins JP, Thompson SG, Deeks JJ, Altman DG. Measuring inconsistency in meta-analyses. BMJ (Clin Res Ed). 2003:327(7414):557-60. 
15. Podhorecka M, Halicka D, Szymczyk A, Macheta A, Chocholska S, Hus M, Darzynkiewicz Z. Assessment of red blood cell distribution width as a prognostic marker in chronic lymphocytic leukemia. Oncotarget. 2016;7(22):32846-53.

16. Iriyama N, Hatta Y, Kobayashi S, Uchino Y, Miura K, Kurita D, Kodaira H, Takahashi H, lizuka Y, Inoue M, et al. Higher red blood cell distribution width is an adverse prognostic factor in chronic-phase chronic myeloid leukemia patients treated with tyrosine kinase inhibitors. Anticancer Res. 2015;35(10):5473-8.

17. Zhou SJ, Fang F, Chen HY, Zhang W, Chen Y, Shi YF, Zheng ZY, Ma YY, Tang $L Y$, Feng JH, et al. Prognostic significance of the red blood cell distribution width in diffuse large B-cell lymphoma patients. Oncotarget. 2017;8(25):40724-31.

18. Perisa V, Zibar L, Sincic-Petricevic J, Knezovic A, Perisa I, Barbic J. Red blood cell distribution width as a simple negative prognostic factor in patients with diffuse large B-cell lymphoma: a retrospective study. Croatian Med J. 2015;56(4):334-43.

19. Luo H, Quan X, Song XY, Zhang L, Yin Y, He Q, Cai S, Li S, Zeng J, Zhang $Q$, et al. Red blood cell distribution width as a predictor of survival in nasal-type, extranodal natural killer/T-cell lymphoma. Oncotarget. 2017;8(54):92522-35.

20. Lee H, Kong SY, Sohn JY, Shim H, Youn HS, Lee S, Kim HJ, Eom HS. Elevated red blood cell distribution width as a simple prognostic factor in patients with symptomatic multiple myeloma. BioMed Res Int. 2014;2014:145619.

21. Wang J, Xie X, Cheng F, Zhou X, Xia J, Qian XF, Wang LL, Guo HF. Evaluation of pretreatment red cell distribution width in patients with multiple myeloma. Cancer Biomarkers. 2017;20(3):267-72.
22. Meng S, Ma Z, Lu C, Liu H, Tu H, Zhang W, Zhou F. Prognostic value of elevated red blood cell distribution width in Chinese patients with multiple myeloma. Ann Clin Lab Sci. 2017;47(3):282-90.

23. Riedl J, Posch F, Konigsbrugge O, Lotsch F, Reitter EM, Eigenbauer E, Marosi C, Schwarzinger I, Zielinski C, Pabinger I, et al. Red cell distribution width and other red blood cell parameters in patients with cancer: association with risk of venous thromboembolism and mortality. PLoS ONE. 2014;9(10):e111440.

24. Yilmaz A, Malya F, Ozturk G, Citgez B, Ozdenkaya Y, Ersavas C, Agan A, Senturk $H$, Karatepe O. Effect of pre-operative red blood cell distribution on cancer stage and morbidity rate in patients with pancreatic cancer. Int J Clin Exp Med. 2014;7(9):3072-5.

25. Lippi G, Targher G, Montagnana M, Salvagno GL, Zoppini G, Guidi GC. Relation between red blood cell distribution width and inflammatory biomarkers in a large cohort of unselected outpatients. Arch Pathol Lab Med. 2009;133(4):628-32.

26. Demirkol S, Balta S, Cakar M, Unlu M, Arslan Z, Kucuk U. Red cell distribution width: a novel inflammatory marker in clinical practice. Cardiol J. 2013;20(2):209.

27. Maccio A, Madeddu C, Gramignano G, Mulas C, Tanca L, Cherchi MC, Floris C, Omoto I, Barracca A, Ganz T. The role of inflammation, iron, and nutritional status in cancer-related anemia: results of a large, prospective, observational study. Haematologica. 2015;100(1):124-32.
Ready to submit your research? Choose BMC and benefit from:

- fast, convenient online submission

- thorough peer review by experienced researchers in your field

- rapid publication on acceptance

- support for research data, including large and complex data types

- gold Open Access which fosters wider collaboration and increased citations

- maximum visibility for your research: over 100M website views per year

At BMC, research is always in progress.

Learn more biomedcentral.com/submissions 